\title{
Room for improvement: Patterns of referral misdiagnosis to a vascular anomalies center*
}

\author{
Daniel E. Levin, Allison L. Speer, James R. Pierce, Donna Nowicki, Alex Arkader, Philip Stanley, \\ Andre Panossian, Dean M. Anselmo \\ Vascular Anomalies Center, Children's Hospital Los Angeles, University of Southern California, Los Angeles, USA \\ Email: dlevin82@gmail.com
}

Received 30 September 2013; revised 26 October 2013; accepted 3 November 2013

Copyright (C) 2013 Daniel E. Levin et al. This is an open access article distributed under the Creative Commons Attribution License, which permits unrestricted use, distribution, and reproduction in any medium, provided the original work is properly cited.

\begin{abstract}
Objective: Vascular anomalies are a diagnostic and therapeutic challenge. Errors in diagnosis lead to treatment delay, inappropriate interventions and prolonged suffering. The aim of this study was to analyze patterns of misdiagnosis among patients referred to a vascular anomalies center (VAC). This will better define the problem and may be used to refine and improve referral guidelines for patients with vascular anomalies. Patients and Methods: After IRB approval, we performed a single-center retrospective review of all patients referred to a vascular anomaly between January 1, 2008 and December 15, 2011. Evaluation of both referral and final diagnosis was made. Data regarding accuracy of diagnosis were determined and compared for both vascular tumors and malformations. Results: Mean age was $7.9 \pm 7.7$ (13 days - 66 years). $42 \%$ had a correct diagnosis at the time of referral. Vascular tumors were correctly diagnosed more often than vascular malformations (58\% vs $38 \%)$. The most common misdiagnosis for infantile hemangioma (IH) was venous malformation (VM). The most common misdiagnosis for VM was IH. Nonspecific and historical terms such as "mass", "lymphangioma", and "cavernous hemangioma" frequently appear as the referral diagnosis. Conclusion: Referral misdiagnosis is common. IH and VM are frequently confused and if there is any uncertainty in the diagnosis, these patients should also be referred to a VAC, in addition to the more complicated anomalies. Outdated nomenclature remains prevalent and continued efforts should be made to adhere to International Society for the Study of Vascular Anomalies (ISSVA) classification. Improvements
\end{abstract}

*Disclosures: the authors report no conflict of interests or financial disclosures. in diagnostic accuracy are likely to greatly improve patients' care.

Keywords: Vascular Anomaly; Misdiagnosis; Venous Malformation; Infantile Hemangioma; Arteriovenous Malformation; Vascular Anomalies Center

\section{INTRODUCTION}

The International Society for the Study of Vascular Anomalies (ISSVA) was established in 1992. Four years later, they adopted a classification system in an effort to accurately diagnose and standardize the nomenclature used to describe vascular anomalies [1]. This classification system was based on the seminal work performed by Mulliken and Glowacki who investigated the endothelial characteristics of vascular anomalies in infants and children [2]. Today, vascular anomalies are divided into two broad categories: vascular tumors and vascular malformations. The pathophysiology, clinical characteristics and biologic behavior of vascular tumors are markedly different from those of vascular malformations. Vascular tumors are generally benign lesions that exhibit hyperplastic proliferation and involution in comparison with vascular malformations which are defects in vascular morphogenesis and tend to grow with the child [1].

Examples of vascular tumors include infantile hemangiomas, congenital hemangiomas (rapidly involuting and non-involuting), tufted angioma and Kaposiform hemangioendothelioma. In addition, vascular malformations are further categorized along rheological characteristics (fast versus slow-flow) as well as the type of anomalous vascular channel. These include capillary malformation (CM), venous malformation (VM), lymphatic malformation (LM) and arteriovenous malformation (AVM) [3]. Moreover, some patients are diagnosed with complex malformations comprised of multiple abnormal channels such as capillary-venous malformation (CVM) and cap- 
illary-lymphatic-venous malformation (CLVM). Accurate diagnosis is made more challenging by the existence of several eponyms associated with vascular anomalies including Klippel-Trenaunay syndrome, Sturge-Weber syndrome and Bannayan-Riley-Ruvalcaba syndrome.

Making the correct diagnosis of a vascular anomaly can be a challenge to providers who do not routinely treat these entities. In addition, the persistence of outdated and historical terms such as, "port wine stain", "strawberry hemangioma" and "cavernous hemangioma" perpetuate diagnostic inaccuracy. In fact, misused terms are frequently found in the published literature. A recent review found that the term "hemangioma" was inaccurate in over $70 \%$ of articles published in 2009 [4]. In 2011, Green et al. demonstrated that among referrals to their vascular anomalies center, $29.6 \%$ of vascular tumors and $54.4 \%$ of vascular malformations were misdiagnosed. Incorrectly diagnosed patients are far more likely to receive misguided treatment [4]. For this reason, our study seeks to delineate patterns of misdiagnosis to our vascular anomalies center (VAC) in an effort to assist referring providers in identifying vascular anomalies accurately and avoiding inappropriate interventions.

\section{PATIENTS AND METHODS}

After obtaining Institutional Review Board approval, a retrospective review of the electronic medical database for VAC patients at Children's Hospital Los Angeles was conducted. All patients referred between January 1, 2008 and December 15, 2011 were included. Patients of all ages were included, and demographics were recorded. Patients referred without a diagnosis of a vascular anomaly were excluded.

All patients with referred with a vascular anomaly were evaluated and final diagnosis was compared to the referral diagnosis and vice versa. Final diagnosis was determined by a team of VAC clinicians based on history, physical exam, imaging and/or pathology results. Patients with a vascular tumor were given a diagnosis according to ISSVA classification, which includes: infantile hemangioma $(\mathrm{IH})$, hemangiomatosis, rapidly involuting congenital hemangioma (RICH), non-involuting congenital hemangioma (NICH), hemangioendothelioma, tufted angioma (TA), Kaposiform hemangioendothelioma (KHE).

All patients with a "vascular malformation" were also evaluated. These included patients with arteriovenous malformation (AVM), capillary malformation (CM), lymphatic malformation (LM), venous malformation (VM), capillary-lymphatic malformation (CLM), capillary-venous malformation (CVM), lymphatic-venous malformation (LVM), or capillary-lymphatic-venous malformation (CLVM). In addition, patients with named syndromes were evaluated including those with Klippel-Trenaunay syndrome (KTS), Proteus syndrome, Bannayan-RileyRuvalcaba syndrome (BRR), Blue-Rubber-Bleb-Nevus syndrome (BRBN), Parkes-Weber syndrome, Cowden syndrome, Sturge Weber (SW) and Gorham-Stout disease (GS).

In addition, data regarding accuracy of diagnosis was determined and compared between fast-flow and slowflow anomalies. Patients referred with a diagnosis not accepted by ISSVA classification were recorded and compared to their most common final diagnosis.

\section{RESULTS}

A total of 331 patients were referred to the Vascular Anomalies Center for evaluation during the 3-year review period. Thirty-nine patients were excluded who were referred without a diagnosis of a vascular anomaly. The charts of 292 patients with a vascular anomaly were evaluated. Average age was 7.9 years (range 13 days - 66 years). The male to female ratio was 0.9. Of the 292 vascular anomalies, $42 \%$ were correctly diagnosed at the time of referral. Fifty of the $292(17 \%)$ had a final diagnosis of a vascular tumor, of which, $58 \%$ were correctly identified. The remaining 242 patients $(83 \%)$ had a final diagnosis of vascular malformation, of which $38 \%$ were diagnosed correctly (see Figure 1).

\subsection{Final Diagnosis of Vascular Tumor}

Infantile hemangioma was the most common VT encountered; $23 \%$ were misdiagnosed by the referring provider. The most common referral misdiagnosis was VM. All patients with hemangiomatosis (defined as $>3 \mathrm{IH}$ ) were correctly diagnosed. Two of the three patients with hemangioendothelioma (1 TA and 2 KHE) were misdiagnosed (see Table 1).

Table 1. Evaluation of vascular anomaly final diagnosis in comparison to the most common referral misdiagnosis.

\begin{tabular}{cc}
\hline $\begin{array}{c}\text { Vascular Anomaly } \\
\text { Final Diagnosis }\end{array}$ & $\begin{array}{c}\text { Most Common Referral } \\
\text { Misdiagnosis }\end{array}$ \\
\hline Vascular Tumors & \\
Infantile Hemangioma & Venous Malformation \\
Congenital Hemangioma & Infantile Hemangioma \\
Hemangioendothelioma & Infantile Hemangioma \\
Vascular Malformations & \\
Venous Malformation & Infantile Hemangioma \\
Lymphatic Malformation & Venous Malformation \\
Capillary Malformation & Klippel-Trenaunay Syndrome \\
Arterial Malformation & Venous Malformation \\
Klippel-Trenaunay Syndrome & Congenital Lymphedema \\
Proteus Syndrome & Capillary Malformation \\
\hline
\end{tabular}




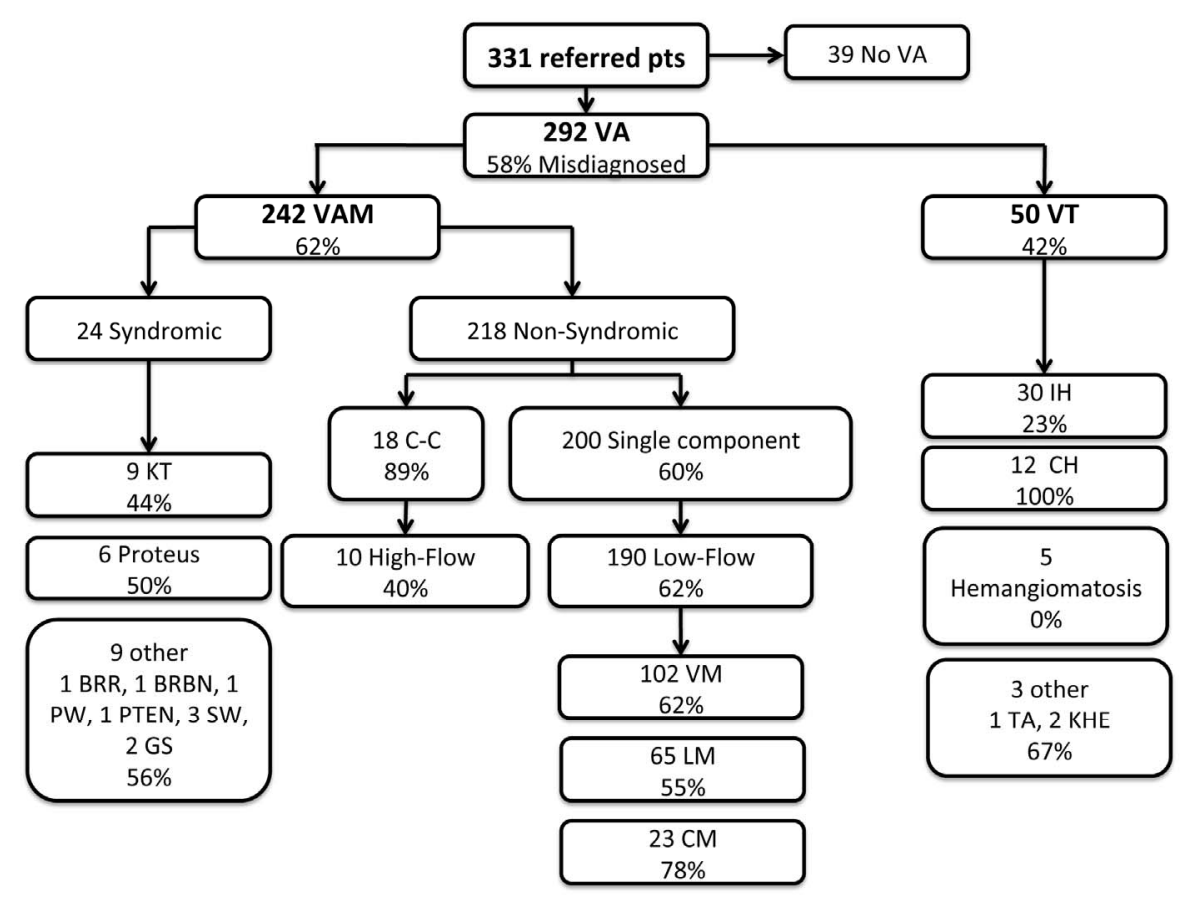

Figure 1. Patterns of misdiagnosis. Boxes list the final diagnosis, number of patients with that diagnosis and percent misdiagnosed. VA $=$ Vascular Anomaly, VAM = Vascular Malformation, VT = Vascular Tumor, VM = Venous Malformation, LM = Lymphatic Malformation, $\mathrm{CM}=$ Capillary Malformation, $\mathrm{IH}=$ Infantile Hemangioma, $\mathrm{CH}=$ Congenital Hemangioma, Hemangiomatosis $\geq 3 \mathrm{IH}$, TA $=$ Tufted Angioma, KHE = Kaposiform Hemangioendothelioma, $\mathrm{C}-\mathrm{C}=$ Complex-Combined, $\mathrm{KT}=$ Klippel Trenaunay, BRR $=$ Bannayan-Riley-Ruvalcaba, BRBN = Blue-Rubber-Bleb-Nevus, PW = Parkes Weber, GS = Gorham-Stout, PTEN = Phosphatase and tensin homolog hamartoma tumor syndrome.

\subsection{Final Diagnosis of Vascular Malformation, Non-Syndromic}

The non-syndromic vascular malformations were divided into two groups, single component $(92 \%)$ and complex-combined (8\%). Sixty percent of the single component malformations and $89 \%$ of the complex-combined malformations were incorrectly diagnosed on referral. Furthermore, the single component malformations were further divided into low-flow (95\%) and high-flow (5\%) malformations. Sixty-two percent of low-flow lesions were misdiagnosed and $40 \%$ of high flow lesions were classified incorrectly. The most common referral misdiagnoses are listed in Table $\mathbf{1 .}$

\subsection{Final Diagnosis of Vascular Malformations Syndromic}

Of the 242 vascular malformations, $10 \%$ (24) had a final diagnosis of a syndrome with associated malformation; $50 \%$ were misdiagnosed. Specific diagnosis is listed in Figure 1.

\subsection{Referral Diagnosis}

The referral diagnosis varied greatly among the patients reviewed. The two most common referral diagnoses were VM and IH. Sixteen percent (47 of 292) of the patients were referred with a VM. Of these patients, $28 \%$ (13 of 47) were incorrectly identified with the most common final diagnosis being LM. Sixteen percent (47 of 292) of the patients were referred with $\mathrm{IH}$. Of these patients, $49 \%$ (23 of 47 ) were misdiagnosed with the most common final diagnosis being VM.

A certain percentage of patients were referred simply as "vascular anomaly" (7\%) or "vascular malformation" $(13 \%)$. Of the patients evaluated for an unspecified "vascular anomaly", the most common final diagnosis was VM and IH. Among patients referred for "vascular malformation", the two most common final diagnoses were VM and LM. KTS was also a common referring diagnosis $(7 \%)$, the majority of which were incorrect $(75 \%)$. Of the patients referred with KTS, the most common correct diagnosis was VM and CM. Additional referral diagnosis can be identified in Table 2.

Many patients were referred based on a suspicious physical exam finding, without a diagnosis. For example, the term, "mass", was the reason for referral in 27 patients, representing $9 \%$ of the total referrals and $22 \%$ of the misdiagnosed patients. Of these 27,12 were found to 
Table 2. Patients referred with nomenclature acceptable by ISSVA classification.

\begin{tabular}{|c|c|c|c|}
\hline \multirow{2}{*}{$\begin{array}{c}\text { Referral Diagnosis (n) } \\
\text { Infantile Hemangioma (47) }\end{array}$} & \multirow{2}{*}{$\begin{array}{c}\text { Misdx (n) } \\
23\end{array}$} & \multicolumn{2}{|c|}{$\begin{array}{l}\text { Top } 2 \text { Most } \\
\text { Common Final } \\
\text { Diagnosis (n) }\end{array}$} \\
\hline & & VM (11) & $\mathrm{NICH}(4)$ \\
\hline Venous Malformation (47) & 13 & LM (6) & $\operatorname{BRR}(1)$ \\
\hline "Vascular Malformation" (37) & 37 & VM (19) & $\operatorname{LM}(7)$ \\
\hline Klippel-Trenaunay (20) & 15 & VM (6) & $\mathrm{CM}(6)$ \\
\hline "Vascular Anomaly" (19) & 19 & $\mathrm{VM}(8)$ & $\mathrm{IH}(4)$ \\
\hline $\begin{array}{c}\text { Lymphatic } \\
\text { Malformation (18) }\end{array}$ & 5 & CLM (3) & VM (2) \\
\hline $\begin{array}{c}\text { Arteriovenous } \\
\text { Malformation (13) }\end{array}$ & 8 & $\mathrm{VM}(4)$ & CVM (1) \\
\hline Capillary Malformation (9) & 3 & CLM (2) & Proteus (1) \\
\hline $\begin{array}{l}\text { Lymphatic Venous } \\
\text { Malformation (2) }\end{array}$ & 2 & VM (2) & \\
\hline PTEN Mutation Syndrome (1) & 1 & PW (1) & \\
\hline Sturge-Weber (2) & 1 & $\mathrm{CM}(1)$ & \\
\hline $\begin{array}{l}\text { Gorham-Stout (3), } \\
\text { CVM (1), BRBN (1), } \\
\text { Hemangiomatosis (3), } \\
\text { KHE, Proteus (2) }\end{array}$ & 0 & & \\
\hline
\end{tabular}

$\mathrm{VA}=$ Vascular Anomaly, VAM = Vascular Malformation, $\mathrm{VT}=$ Vascular Tumor, $\mathrm{VM}=$ Venous Malformation, $\mathrm{LM}=$ Lymphatic Malformation, $\mathrm{CM}$ = Capillary Malformation, CLM = Capillary Lymphatic Malformation, $\mathrm{CVM}=$ Capillary Venous Malformation, $\mathrm{IH}=$ Infantile Hemangioma, $\mathrm{CH}=$ Congenital Hemangioma, Hemangiomatosis $\geq 3 \mathrm{IH}, \mathrm{TA}=$ Tufted Angioma, $\mathrm{KHE}=$ Kaposiform Hemangioendothelioma, C-C $=$ Complex-Combined, $\mathrm{KT}=$ Klippel Trenaunay, BRR = Bannayan-Riley-Ruvalcaba, BRBN = Blue-Rubber-Bleb-Nevus, PW = Parkes Weber, GS = Gorham-Stout, PTEN $=$ Phosphatase and tensin homolog hamartoma tumor syndrome, Misdx $=$ Misdiagnosis.

have a LM, 9 had a VM, 3 had an AVM and the other 3 were diagnosed with a NICH, CLM and IH, respectively.

Outdated and historical terms and diagnoses were frequently used by referring providers and were considered a misdiagnosis. Fifteen patients were evaluated for a "lymphangioma", representing $12 \%$ of all misdiagnosed patients. Of these, 12 had a final diagnosis of LM, 2 VM and 1 CLM.

"Cavernous hemangioma" was used to describe 4 anomalies, 3 of which were diagnosed with a VM and 1 with an IH. Three patients were referred for "cystic hygroma" with a final diagnosis of LM in 2 and LVM in 1. Additionally, 3 patients were referred for "port wine stain" and 2 were referred for "intramuscular hemangioma". No patients were referred with a diagnosis of "strawberry hemangioma". Table 3 lists those patients referred with a diagnosis not included in the ISSVA classification system.
Table 3. Patients with a referral diagnosis that used nonISSVA nomenclature in comparison to the most commonly encountered correct final diagnosis.

\begin{tabular}{ccc}
\hline Referral Diagnosis (n) & \multicolumn{2}{c}{ Top 2 Most Common } \\
Final Diagnosis (n)
\end{tabular}

$\mathrm{VM}=$ Venous Malformation, $\mathrm{LM}=$ Lymphatic Malformation, $\mathrm{CM}=$ Capillary Malformation, $\mathrm{IH}=$ Infantile Hemangioma, $\mathrm{KT}=$ Klippel Trenaunay, $\mathrm{SW}=$ Sturge Weber.

\section{DISCUSSION}

30 years have transpired since Mulliken and Glowacki first published their recommendations for the classification of vascular anomalies [2] and 17 years since ISSVA adapted these recommendations into the current accepted classification system [1]. Nevertheless, confusing nomenclature and the persistence of outdated terminology continues to yield frequent diagnostic inaccuracies and delays in treatment [5]. To help combat this problem, a number of multidisciplinary vascular anomalies centers (VAC) have been established across the United States to optimize the care of this challenging patient population. In 2011, Greene et al., established improved guidelines for referral [6]. In doing so, they also confirmed the frequent inaccuracies in referral nomenclature.

Overall, $58 \%$ of vascular anomalies treated in our $\mathrm{VAC}$ were found to have an incorrect diagnosis at the time of referral. When evaluated based on ISSVA classification, $42 \%$ of tumors and $62 \%$ of malformations were misdiagnosed. This is similar to the findings of Greene $e t$ al., who found that $30 \%$ of tumors and $54 \%$ of malformations were misdiagnosed [6]. This high degree of inaccuracy underscores the need for specialized VAC and the call for greater understanding of patterns of misdiagnosis.

Infantile hemangiomas were misdiagnosed in $23 \%$ of patients referred to our VAC. Conversely, $62 \%$ of patients with VMs were erroneously identified by the referring provider. Interestingly, the most common referral misdiagnosis for an IH was VM and for a VM was an IH. It becomes evident that referring physicians frequently confuse these two very distinct vascular anomalies (see 
Figures 2 and 3). Generally speaking, VM should be distinguishable from IH on the basis of a thorough history and physical exam and an understanding of the pathogenesis of these lesions. Therefore, one may surmise that improving provider education on methods of differentiating IH from VM has the potential to greatly influence diagnostic accuracy and treatment.

In addition, when the final diagnosis was a complexcombined anomaly, the referring physician frequently identified at least one component, but missed the others. One potential explanation is that referring clinicians are unaware of the existence of combined vascular malfor-

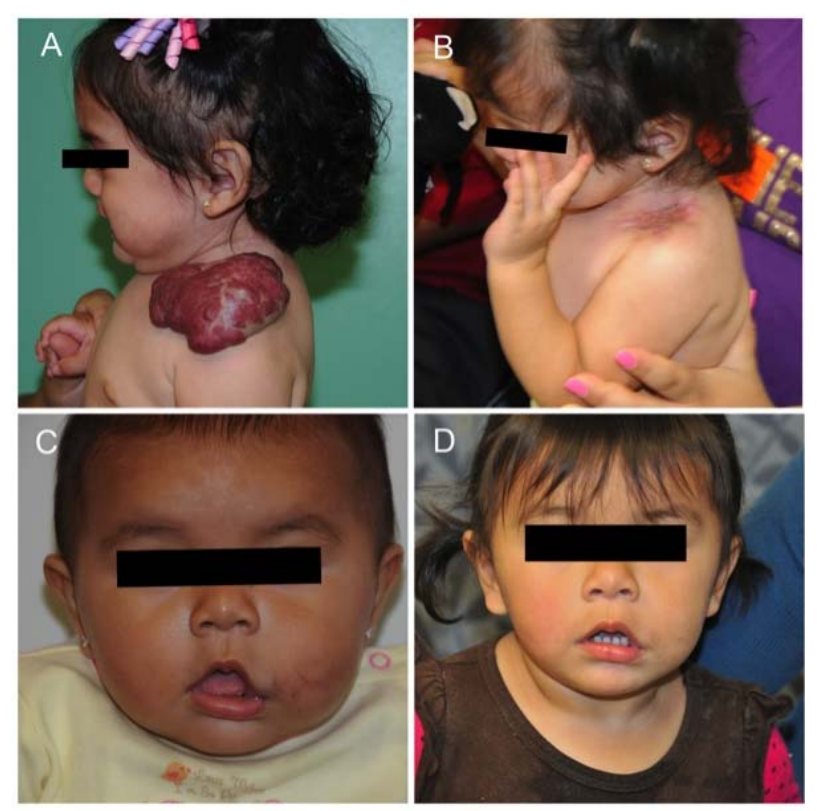

Figure 2. Clinical presentation of infantile hemangioma (IH) may vary greatly from patient to patient. Typical history includes a period of rapid proliferation during the first several months of life, followed by a period of involution beginning at 1 year of age and continuing for a variable length of time. Spontaneous regression frequently occurs, but treatments exist for complicated cases. Panel (A) demonstrates a large, dome shaped, lobulated shoulder IH (A) that ultimately required surgical removal (B) after failure to spontaneously involute or respond to beta blocker and medical therapy. Panel (C) demonstrates and IH that spontaneously involution (D).
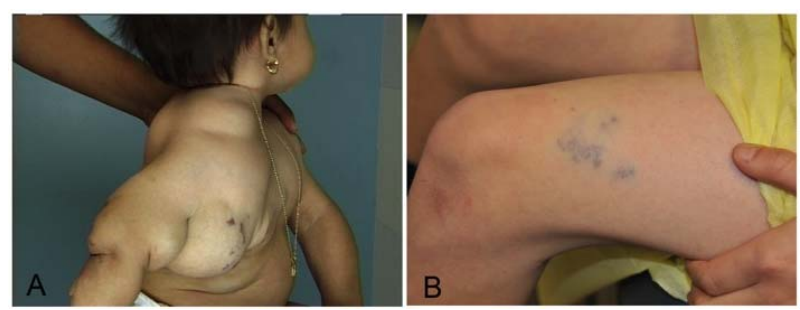

Figure 3. Venous malformations may present as large, disfiguring malformations (A) or as a vague blue patch (B). Unlike $\mathrm{IH}$, these do not regress and treatment is often multimodal with medical, sclerotherapy, laser or surgical techniques. mations, therefore, concluding their diagnostic evaluation once a single component was identified.

This is further substantiated by the fact that when evaluating patients based on referral diagnosis, complex combined malformations were only mentioned 3 times, whereas 18 patients had a final diagnosis consistent with complex combined malformation. From this, we encourage providers to consider the possibility of a combined lesion when assessing an anomaly and to take steps, when needed, to evaluate for additional components.

Adherence to correct nomenclature is not just a matter of semantics. These classifications are based on marked histopathologic differences [7]. Patients that carry the wrong diagnosis are subject to inappropriate treatment and prolonged delays in management [4]. Therefore, the use of terms such as, "cavernous hemangioma" and "lymphangioma" will likely lead to a detrimental effect on patients. In our review, referring physicians were less commonly using such phrases. For example, "cavernous hemangioma" appeared just 4 times during the 3-year referral period (most frequent final diagnosis was VM) and none of our patients were labeled as having a "strawberry hemangioma". Perhaps community patient care providers are beginning to limit their use of these and other ill-described terms (e.g., "port wine stain" or "cystic hygroma").

Unfortunately, however, other outdated terminology still persists in the referral population. For example, among all the patients who were misdiagnosed, $12 \%$ were referred with a diagnosis of "lymphangioma". The majority of these patients were subsequently diagnosed with LM, suggesting the referring providers are unaware of the correct ISSVA nomenclature. Perhaps more concerning was the frequent use of the term "mass", as a referring diagnosis. Among all misdiagnosed patients, $22 \%$ were referred for a mass on physical exam without any further classification. The reluctance to provide the patient with a true diagnosis is likely related, in part, to the complexity of diagnosing vascular anomalies.

We found considerable variability with patients diagnosed with syndromic conditions associated with vascular malformations. For example, of the 7 patients with a final diagnosis of KT, 4 were misdiagnosed on referral.

However, KT was the referring diagnosis for 20 patients, which was incorrect for 15 patients. Many patients were diagnosed with $\mathrm{KT}$ based on the presence of a malformation with associated limb hypertrophy.

This study has several limitations. Although this VAC has a broad geographic referral base, it is unclear if the patterns we describe would be consistent across the country. In addition, there are a number of very rare conditions mentioned in this review that were observed infrequently, thereby limiting the conclusions that can be made regarding these rare conditions. Furthermore, al- 
though we have better defined the problem, we have not tested an intervention that may be guided to improve diagnostic accuracy among referring physicians. This is an ideal opportunity for future research in quality improvement.

\section{CONCLUSION}

Overall, we have identified a number of patterns of misdiagnosis that should aid both the referring clinician and VAC provider in avoiding some of the pitfalls and errors in diagnosis. Ideally, expanding education of referring providers as well as strictly adhering to the internationally accepted ISSVA guidelines will improve rates of proper diagnosis most reliably. It is the responsibility of VAC clinicians to shine a spotlight on this pervasive problem in one of the newest areas of clinical medicine.

\section{REFERENCES}

[1] Enjolras, O., Wassef, M. and Chapot, R. (2007) Color atlas of vascular tumors and vascular malformations. Cambridge University Press, New York.

[2] Mulliken, J.B. and Glowacki, J. (1982) Hemangiomas and vascular malformations in infants and children: A classification based on endothelial characteristics. Plastic and Reconstructive Surgery, 69, 412-422. http://dx.doi.org/10.1097/00006534-198203000-00002

[3] Enjolras, O. and Mulliken, J.B. (1997) Vascular tumors and vascular malformations (new issues). Advanced Dermatology, 13, 375-423.

[4] Hassanein, A.H., Mulliken, J.B., Fishman, S.J. and Greene, A.K. (2011) Evaluation of terminology for vascular anomalies in current literature. Plastic and Reconstructive Surgery, 127, 347-351. http://dx.doi.org/10.1097/PRS.0b013e3181f95b83

[5] Marler, J.J. and Mulliken, J.B. (2005) Current management of hemangiomas and vascular malformations. Clinics in Plastic Surgery, 32, 99-116, ix. http://dx.doi.org/10.1016/j.cps.2004.10.001

[6] Greene, A.K., Liu, A.S., Mulliken, J.B., Chalache, K. and Fishman, S.J. (2011) Vascular anomalies in 5621 patients: Guidelines for referral. Journal of Pediatric Surgery, 46, 1784-1789.

http://dx.doi.org/10.1016/j.jpedsurg.2011.05.006

[7] Aboutalebi, A., Jessup, C.J., North, P.E. and Mihm, M.C. (2012) Histopathology of vascular anomalies. Facial Plastic Surgery, 28, 545-553. http://dx.doi.org/10.1055/s-0032-1329929 